\title{
Optimal shape and location of sensors or actuators in PDE models
}

\author{
Yannick Privat $^{1}$, Emmanuel Trélat $^{2}$, Enrique Zuazua $^{3}$
}

\begin{abstract}
We investigate the problem of optimizing the shape and location of sensors and actuators for evolution systems driven by distributed parameter systems or partial differential equations (PDE), like for instance the wave equation, the Schrödinger equation, or a parabolic system, on an arbitrary domain $\Omega$, in any space dimension, and with suitable boundary conditions if there is a boundary, which can be of Dirichlet, Neumann, mixed or Robin. This kind of problem is frequently encountered in applications where one aims, for instance, at maximizing the quality of reconstruction of the solution, using only a partial observation. From the mathematical point of view, using probabilistic considerations we model this problem as that of maximizing the so-called randomized observability constant, over all possible subdomains of $\Omega$ having a prescribed measure. The spectral analysis of this problem reveals intimate connections with the theory of quantum chaos. More precisely, we provide a solution to this problem when the domain $\Omega$ satisfies suitable quantum ergodicity assumptions.
\end{abstract}

\section{INTRODUCTION}

Our objective is to model and solve the problem of optimizing the shape and location of sensors and actuators for processes modeled by a PDE on any open bounded connected subset of a Riemannian manifold, with various possible boundary conditions. Unexpectedly, our results reveal intimate connections with quantum ergodicity issues. Less surprisingly, our results strongly depend on the nature of the PDE under consideration.

\section{A. The context}

The literature on optimal observation or sensor location problems is abundant in engineering applications (see, e.g., [3], [8], [11], [12], [20], [22], [23], [24] and references therein), where the aim is often to optimize the number, the place and the type of sensors in order to improve the estimation of the state of the system. Fields of applications are very numerous and concern for example active structural acoustics, piezoelectric actuators, vibration control in mechanical structures, damage detection and chemical reactions, just to name a few of them. In most of these applications the method consists in approximating appropriately the problem by selecting a finite number of possible optimal candidates

\footnotetext{
${ }^{1}$ Yannick Privat is with CNRS, Université Pierre et Marie Curie (Univ. Paris 6), UMR 7598, Laboratoire Jacques-Louis Lions, F-75005, Paris, France yannick.privateupme. fr

${ }^{2}$ Emmanuel Trélat is with Université Pierre et Marie Curie (Univ. Paris 6) and Institut Universitaire de France and Team GECO Inria Saclay, CNRS UMR 7598, Laboratoire Jacques-Louis Lions, F-75005, Paris, France emmanuel.trelateupmc. fr

${ }^{3}$ Enrique Zuazua is with BCAM - Basque Center for Applied Mathematics, Mazarredo, 14 E-48009 Bilbao-Basque Country-Spain, and Ikerbasque, Basque Foundation for Science, Alameda Urquijo 36-5, Plaza Bizkaia, 48011, Bilbao-Basque Country-Spain zuazua@bcamath.org
}

and of recasting the problem as a finite-dimensional combinatorial optimization problem. In many of these contributions the sensors have a prescribed shape (for instance, balls with a prescribed radius) and then the problem consists of placing optimally a finite number of points (the centers of the balls) and thus is finite-dimensional, since the class of optimal designs is replaced with a compact finite-dimensional set.

In the present paper our objective is to run the search over all possible subsets, in other words we optimize not only the placement but also the shape of the sensors or actuators.

Among the existing approaches, the closest one to ours consists of considering truncations of Fourier expansion representations. Adopting such a Fourier point of view, the authors of [9], [10] studied optimal stabilization issues of the one-dimensional wave equation and, up to our knowledge, these are the first articles in which one can find characterizations of the optimal set whenever it exists, for the problem of determining the best possible shape and position of the damping subdomain of a given measure. In [5] the authors investigate the problem modeled in [20] of finding the best possible distributions of two materials (with different elastic Young modulus and different density) in a rod in order to minimize the vibration energy in the structure. For this optimal design problem in wave propagation, the authors of [5] prove existence results and provide convexification and optimality conditions. The authors of [1] also propose a convexification formulation of eigenfrequency optimization problems applied to optimal design. In [7] the authors discuss several possible criteria for optimizing the damping of abstract wave equations and derive optimality conditions for a certain criterion related to a Lyapunov equation.

From the mathematical point of view, the issue of studying a relaxed version of optimal design problems for the shape and position of sensors or actuators has been investigated in a series of articles. In [14], the authors study a convexified version of the optimal location of controllers for the heat equation problem, noticing that such problems are often illposed. In [2], the authors consider a similar problem and study the asymptotic behavior as the final time $T$ goes to infinity of the solutions of the relaxed problem; they prove that optimal designs converge to an optimal relaxed design of the corresponding two-phase optimization problem for the stationary heat equation. We also mention [13] where, for fixed initial data, numerical investigations are used to provide evidence that the optimal location of null-controllers of the heat equation problem is an ill-posed problem. In [18] we proved that, for fixed initial data as well, the problem of optimal shape and location of sensors is always well-posed for heat, wave or Schrödinger equations, and we solved it, 
showing that the complexity of the optimal set depends on the regularity of the initial data; in particular we showed that, even for smooth initial data the optimal set can be fractal.

A huge difference between these works and the approach developed here is in the fact that all criteria introduced in the sequel take into consideration all possible initial data, and moreover the optimization will run over all possible measurable subsets in $\mathcal{U}_{L}$. It is the idea developed in [16], [15], [17], [19] where the problem of the optimal location of an observation subset $\omega$ among all possible subsets of a given measure or volume fraction of $\Omega$ was addressed and solved for wave and Schrödinger equations and also for general parabolic equations. A relevant spectral criterion, viewed as a measure of eigenfunction concentration and not depending on the initial conditions was considered, in order to design an optimal observation or control set in an uniform way, independent of the data and solutions under consideration, as explained next. Such a kind of uniform criterion was earlier introduced for the one-dimensional wave equation in [9], [10] in view of investigating optimal stabilization issues.

\section{B. Problem formulation}

To begin with, let us focus on a particular case of our study, starting from a practical problem. Assume that $\Omega$ is a given bounded open subset of $\mathbb{R}^{n}$, representing for instance a cavity in which some signals are propagating according to the wave equation

$$
\partial_{t t} y=\triangle y
$$

with Dirichlet boundary conditions. Having for example in mind some reconstruction inverse problem, assume that one is allowed to place some sensors in the cavity, in order to make some measurements of the signals propagating in $\Omega$ over a certain horizon of time. We assume that we have the choice not only of the placement of the sensors but also of their shape. Let us address the question of knowing what is the best possible shape and location of sensors, achieving the best possible observation, in some sense to be made precise. This problem of optimal observability, inspired by control theoretical considerations, is intimately related to those of optimal controllability and stabilization.

At this step, the question is too much informal and a first challenge is to settle it properly in the mathematical world, so that the resulting problem will be both mathematically solvable and relevant in view of practical issues.

A first obvious but important remark is that, for any problem consisting of optimizing the observation, certainly the best policy consists of observing the solutions over the whole domain $\Omega$. This is however clearly not reasonable and in practice the domain covered by sensors is limited, due for instance to cost considerations. From the mathematical point of view, we model this basic limitation by considering as the set of unknowns, the set of all possible measurable subsets $\omega$ of $\Omega$ that are of Lebesgue measure $|\omega|=L|\Omega|$, where $L \in(0,1)$ is some fixed real number. Any such subset represents the sensors put in $\Omega$, and we assume that we are able to measure the restrictions of the solutions of (1) to $\omega$.

\section{MODELING}

Let us model the notion of best observation. For all $\left(y^{0}, y^{1}\right) \in L^{2}(\Omega, \mathbb{C}) \times H^{-1}(\Omega, \mathbb{C})$, there exists a unique solution $y \in C^{0}\left(0, T ; L^{2}(\Omega, \mathbb{C})\right) \cap C^{1}\left(0, T ; H^{-1}(\Omega, \mathbb{C})\right)$ of (1) such that $y(0, \cdot)=y^{0}(\cdot)$ and $y_{t}(0, \cdot)=y^{1}(\cdot)$. Let $T>0$. We say that (1) is observable on $\omega$ in time $T$ if there exists $C>0$ such that

$$
C\left\|\left(y^{0}, y^{1}\right)\right\|_{L^{2} \times H^{-1}}^{2} \leqslant \int_{0}^{T} \int_{\omega}|y(t, x)|^{2} d x d t,
$$

for all $\left(y^{0}, y^{1}\right) \in L^{2}(\Omega, \mathbb{C}) \times H^{-1}(\Omega, \mathbb{C})$. This inequality is called an observability inequality, and is of great importance in view of showing the well-posedness of some inverse problems. It is well known that within the class of $\mathcal{C}^{\infty}$ domains $\Omega$, this observability property holds if the pair $(\omega, T)$ satisfies the Geometric Control Condition in $\Omega$ (see [4]), according to which every ray of geometrical optics that propagates in the cavity $\Omega$ and is reflected on its boundary $\partial \Omega$ intersects $\omega$ within time $T$. The observability constant $C_{T}\left(\chi_{\omega}\right)$ is defined as the infimum of the quantities

$$
\int_{0}^{T} \int_{\Omega} \chi_{\omega}(x)|y(t, x)|^{2} d x d t /\left\|\left(y^{0}, y^{1}\right)\right\|_{L^{2} \times H^{-1}}^{2}
$$

over all initial data $\left(y^{0}, y^{1}\right) \in L^{2}(\Omega, \mathbb{C}) \times H^{-1}(\Omega, \mathbb{C}) \backslash$ $\{(0,0)\}$. It is the largest possible constant for which (2) holds. It depends both on the time $T$ (the horizon time of observation) and on the subset $\omega$ on which the measurements are done.

A priori, it might appear natural to model the problem of best observability as that of maximizing the functional $\chi_{\omega} \mapsto C_{T}\left(\chi_{\omega}\right)$ over the set

$$
\mathcal{U}_{L}=\left\{\chi_{\omega} \mid \omega \subset \Omega \text { measurable, }|\omega|=L|\Omega|\right\} .
$$

However, this choice of model leads to a mathematical problem that is difficult to handle from the theoretical point of view, and more importantly, it is not relevant in view of practical issues. Let us explain these two difficulties.

First of all, a spectral expansion of the solutions shows the emergence of crossed terms that are difficult to treat. Indeed, let $\left(\phi_{j}\right)_{j \in \mathbf{N}^{*}}$ be a Hilbert basis of $L^{2}(\Omega)$ consisting of eigenfunctions of the Dirichlet-Laplacian operator on $\Omega$, associated with the negative eigenvalues $\left(-\lambda_{j}^{2}\right)_{j \in \mathbf{N}^{*}}$. Then any solution $y$ of (1) can be expanded as

$$
y(t, x)=\sum_{j=1}^{+\infty}\left(a_{j} e^{i \lambda_{j} t}+b_{j} e^{-i \lambda_{j} t}\right) \phi_{j}(x),
$$

where the coefficients $a_{j}$ and $b_{j}$ account for initial data. It follows that $2 C_{T}^{(W)}\left(\chi_{\omega}\right)$ is the infimum of the quantities $\int_{0}^{T} \int_{\omega}\left|\sum_{j=1}^{+\infty}\left(a_{j} e^{i \lambda_{j} t}+b_{j} e^{-i \lambda_{j} t}\right) \phi_{j}(x)\right|^{2} d x d t$ over all possible sequences $\left(a_{j}\right)$ and $\left(b_{j}\right)$ of $\ell^{2}(\mathbb{C})$ such that $\sum_{j=1}^{+\infty}\left(\left|a_{j}\right|^{2}+\left|b_{j}\right|^{2}\right)=1$. Then, maximizing this functional over $\mathcal{U}_{L}$ appears to be very difficult from the theoretical point of view, due to the crossed terms $\int_{\omega} \phi_{j} \phi_{k} d x$ measuring the interaction over $\omega$ between distinct eigenfunctions. 
The second difficulty with this model is its lack of relevance in practice. Indeed, the observability constant $C_{T}\left(\chi_{\omega}\right)$ is deterministic and provides an account for the worst possible case. Hence, in this sense, it is a pessimistic constant. In practice when realizing a large number of measures, it may be expected that this worst case does not occur so often, and one would like that the observation be optimal for most of experiments. This leads us to consider rather an averaged version of the observability inequality over random initial data. In few words, we define what we call the randomized observability constant $2 C_{T, \text { rand }}\left(\chi_{\omega}\right)$ as the infimum of the quantities $\mathbb{E} \int_{0}^{T} \int_{\omega}\left|\sum_{j=1}^{+\infty}\left(\beta_{1, j}^{\nu} a_{j} e^{i \lambda_{j} t}+\beta_{2, j}^{\nu} b_{j} e^{-i \lambda_{j} t}\right) \phi_{j}(x)\right|^{2} d x d t$ over all possible sequences $\left(a_{j}\right)$ and $\left(b_{j}\right)$ of $\ell^{2}(\mathbb{C})$ such that $\sum_{j=1}^{+\infty}\left(\left|a_{j}\right|^{2}+\left|b_{j}\right|^{2}\right)=1$, where $\left(\beta_{1, j}^{\nu}\right)_{j \in \mathbb{N}^{*}}$ and $\left(\beta_{2, j}^{\nu}\right)_{j \in \mathbf{N}^{*}}$ are two sequences of (for example) i.i.d. Bernoulli random laws on a probability space $(\mathcal{X}, \mathcal{A}, \mathbb{P})$, and $\mathbb{E}$ is the expectation over the $\mathcal{X}$ with respect to the probability measure $\mathbb{P}$. It corresponds to an averaged version of the observability inequality over random initial data. We refer to [17] for more details and properties of the randomization procedure. The following result gives a characterization of the randomized observability constant.

Theorem 1 ([17]): For every measurable subset $\omega$ of $\Omega$, there holds $2 C_{T \text {,rand }}\left(\chi_{\omega}\right)=T \inf _{j \in \mathbb{N}^{*}} \int_{\omega} \phi_{j}(x)^{2} d x$.

It is interesting to note that there always holds $C_{T}\left(\chi_{\omega}\right) \leqslant$ $C_{T \text {,rand }}\left(\chi_{\omega}\right)$, and that the strict inequality holds for instance in each of the following cases:

- in $1 \mathrm{D}$, with $\Omega=(0, \pi)$ and Dirichlet boundary conditions, whenever $T$ is not an integer multiple of $\pi$;

- in multi-D, with $\Omega$ stadium-shaped, whenever $\omega$ contains an open neighborhood of the wings (in that case there even holds $\left.C_{T}^{(W)}\left(\chi_{\omega}\right)=0\right)$.

Taking into account the fact that, in practice, it is expected that a large number of measurements is to be done, we finally model the problem of best observability in the following more relevant way:

Maximize the functional

$$
J\left(\chi_{\omega}\right)=\inf _{j \in \mathbf{N}^{*}} \int_{\omega} \phi_{j}(x)^{2} d x
$$

over the set $\mathcal{U}_{L}$.

The functional $J$ appears as a criterion giving an account for eigenfunctions concentration properties.

\section{SOLVING}

In view of solving the uniform optimal design problem $\sup _{\chi_{\omega} \in \mathcal{U}_{L}} J\left(\chi_{\omega}\right)$, we first consider a convexified version, by considering the convex closure of the set $\mathcal{U}_{L}$ for the $L^{\infty}$ weak star topology, that is $\overline{\mathcal{U}}_{L}=\{a \in$ $\left.L^{\infty}(\Omega,[0,1])\left|\int_{\Omega} a(x) d x=L\right| \Omega \mid\right\}$. The convexified problem then consists of maximizing the functional $J(a)=$ $\inf _{j \in \mathbf{N}^{*}} \int_{\Omega} a(x) \phi_{j}(x)^{2} d x$ over $\overline{\mathcal{U}}_{L}$. Clearly there exists a maximizer, but since the functional $J$ is not lower semicontinuous it is not clear whether or not there may be a gap between the problem (5) and its convexified version.
The following result shows that under appropriate quantum ergodicity assumptions there is no gap.

Theorem 2 (No-gap, and optimal value of J, see [17]): Assume that there exists a subsequence of the sequence of probability measures $\mu_{j}=\phi_{j}^{2}(x) d x$ converging vaguely to the uniform measure $\frac{1}{|\Omega|} d x$ (Weak Quantum Ergodicity assumption), and that the sequence of eigenfunctions $\phi_{j}$ is uniformly bounded in $L^{\infty}(\Omega)$. Then

$$
\sup _{\chi_{\omega} \in \mathcal{U}_{L}} J\left(\chi_{\omega}\right)=\max _{a \in \overline{\mathcal{U}}_{L}} J(a)=L,
$$

for every $L \in(0,1)$. In other words, there is no gap between the problem (5) and its convexified version.

At this step, it follows from Theorems 1 and 2 that, under quantum ergodicity assumptions, the maximal possible value of $C_{T \text {,rand }}\left(\chi_{\omega}\right)$ (over the set $\mathcal{U}_{L}$ ) is equal to $T L / 2$.

Remark 1: The quantum ergodicity assumptions of the above result hold true in any hypercube with Dirichlet boundary conditions when one considers the usual Hilbert basis made of products of sine functions.

Remark 2: They are sufficient but not necessary to derive such a no-gap statement: indeed we can prove that it still holds true if $\Omega$ is a two-dimensional disk (with the usual eigenfunctions parametrized by Bessel functions), although the eigenfunctions do not equidistribute as the eigenfrequencies increase, as illustrated by the well-known whispering galleries effect.

Remark 3: We are not aware of any example in which there is a gap between the problem (5) and its convexified version. It is interesting to note that, since the spectral functional $J$ defined by (5) depends on the specific choice of the orthonormal basis $\left(\phi_{j}\right)_{j \in \mathbf{N}^{*}}$ of eigenfunctions of the Dirichlet-Laplacian, one can consider an intrinsic version of the problem, consisting of maximizing the spectral functional $J_{\text {int }}\left(\chi_{\omega}\right)=\inf _{\phi \in \mathcal{E}} \int_{\omega} \phi(x)^{2} d x$ over $\mathcal{U}_{L}$, where $\mathcal{E}$ denotes the set of all normalized eigenfunctions of the DirichletLaplacian. For this problem we have a result similar to the one above (see [17]), but we are moreover able to provide an explicit example where a gap occurs between the problem and its convexified formulation, by considering for instance the unit half-sphere with Dirichlet boundary conditions, and certain quantum limits of a Dirac type.

Remark 4: Under the stronger assumption that the whole sequence of $\mu_{j}=\phi_{j}^{2}(x) d x$ converges vaguely to the uniform measure $\frac{1}{|\Omega|} d x$ (Quantum Unique Ergodicity assumption), and assuming that the $\phi_{j}$ 's are uniformly bounded in some $L^{p}(\Omega)$ with $p>2$, we can prove that the supremum of $J$ over the set of Jordan measurable subsets of measure $L|\Omega|$ is equal to $L$. The proof is constructive and builds a maximizing sequence of subsets, showing that it is possible to increase the value of $J$ with subsets having an increasing number of connected components.

Our results eventually show intimate connections between domain optimization and quantum ergodicity properties of $\Omega$. Such a relation was suggested in the early work [6] concerning the exponential decay properties of dissipative wave equations. 


\section{NONEXISTENCE OF AN OPTIMAL SET AND REMEDIES}

The maximum of $J$ over $\overline{\mathcal{U}}_{L}$ is clearly reached (in general, in an infinite number of ways). The question of the reachability of the supremum of $J$ over $\mathcal{U}_{L}$, that is, the existence of an optimal classical set, is a difficult question in general. In particular cases it can however be addressed using harmonic analysis. For instance in dimension one, we can prove that the supremum is reached if and only if $L=1 / 2$ (and there is an infinite number of optimal sets). In higher dimension, the question is completely open, and we conjecture that, for generic domains $\Omega$ and generic values of $L$, the supremum is not reached and hence there does not exist any optimal set. It can however be noted that, in the two-dimensional Euclidean square, if we restrict the search of optimal sets to Cartesian products of $1 \mathrm{D}$ subsets, then the supremum is reached if and only if $L \in\{1 / 4,1 / 2,3 / 4\}$ (see [17]).

In view of that, it is then natural to study a finitedimensional spectral approximation of the problem, namely:

Maximize the functional

$$
J_{N}\left(\chi_{\omega}\right)=\min _{1 \leqslant j \leqslant N} \int_{\omega} \phi_{j}(x)^{2} d x
$$

over the set $\mathcal{U}_{L}$.

The existence and uniqueness of an optimal set $\omega^{N}$ is then not difficult to prove, as well as a $\Gamma$-convergence property of $J_{N}$ towards $J$ for the weak star topology of $L^{\infty}$. Moreover, the sets $\omega^{N}$ have a finite number of connected components, expected to increase in function of $N$. The
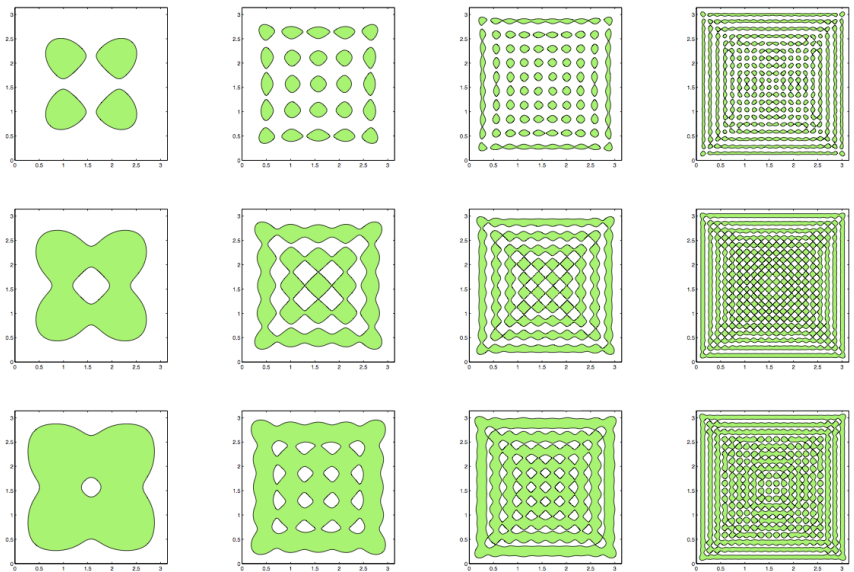

Fig. 1. $\Omega=(0, \pi)^{2}$. Row 1: $L=0.2$; row 2: $L=0.4$; row 3: $L=0.6$. From left to right: $N=2$ (4 eigenmodes), $N=5$ (25 eigenmodes), $N=10$ (100 eigenmodes), $N=20$ (400 eigenmodes). The optimal domain is in green.

numerical simulations of Fig. 1 show the shapes of these sets; their increasing complexity which can be observed as $N$ increases is in accordance with the conjecture of the nonexistence of an optimal set maximizing $J$. It can be noted that, in the one-dimensional case, for $L$ sufficiently small, loosely speaking, the optimal domain $\omega^{N}$ for $N$ modes is the worst possible one when considering the truncated problem with $N+1$ modes (spillover phenomenon; see [10], [15]).

This intrinsic instability is in some sense due to the fact that in the definition of the spectral criterion (5) all modes have the same weight, and the same criticism can be made on the observability inequality (2). Due to the increasing complexity of the geometry of highfrequency eigenfunctions, it could indeed be expected that the optimal shape and placement problem would be complicated. This leads to the intuition that lower frequencies should be more weighted than the higher ones, and then it seems relevant to introduce a weighted version of the observability inequality (2), by considering the (equivalent) inequality

$$
\begin{aligned}
& C_{T, \sigma}\left(\chi_{\omega}\right)\left(\left\|\left(y^{0}, y^{1}\right)\right\|_{L^{2} \times H^{-1}}^{2}+\sigma\left\|y^{0}\right\|_{H^{-1}}^{2}\right) \\
& \leqslant \int_{0}^{T} \int_{\omega}|y(t, x)|^{2} d x d t,
\end{aligned}
$$

where $\sigma \geqslant 0$ is some weight. There holds $C_{T, \sigma}\left(\chi_{\omega}\right) \leqslant$ $C_{T}\left(\chi_{\omega}\right)$, and considering as before an averaged version of this weighted observability inequality over random initial data leads to $2 C_{T, \sigma \text {,rand }}^{(W)}\left(\chi_{\omega}\right)=T J_{\sigma}\left(\chi_{\omega}\right)$, where the weighted spectral criterion $J_{\sigma}$ is defined by

$$
J_{\sigma}\left(\chi_{\omega}\right)=\inf _{j \in \mathbb{N}^{*}} \sigma_{j} \int_{\omega} \phi_{j}(x)^{2} d x,
$$

with $\sigma_{j}=\lambda_{j}^{2} /\left(\sigma+\lambda_{j}^{2}\right)$ (increasing sequence of positive real numbers converging to 1 ; see [17] for details). The truncated criterion $J_{\sigma, N}$ is then defined accordingly, by keeping only the $N$ first modes. We then have the following result.

Theorem 3 ([17]): Assume that the whole sequence of probability measures $\mu_{j}=\phi_{j}^{2}(x) d x$ converges vaguely to the uniform measure $\frac{1}{|\Omega|} d x$ (Quantum Unique Ergodicity assumption), and that the sequence of eigenfunctions $\phi_{j}$ is uniformly bounded in $L^{\infty}(\Omega)$. Then, for every $L \in\left(\sigma_{1}, 1\right)$, there exists $N_{0} \in \mathbf{N}^{*}$ such that

$$
\max _{\chi_{\omega} \in \mathcal{U}_{L}} J_{\sigma}\left(\chi_{\omega}\right)=\max _{\chi_{\omega} \in \mathcal{U}_{L}} J_{\sigma, N}\left(\chi_{\omega}\right) \leqslant \sigma_{1}<L
$$

for every $N \geqslant N_{0}$. In particular, the problem of maximizing $J_{\sigma}$ over $\mathcal{U}_{L}$ has a unique solution $\chi_{\omega^{N_{0}}}$, and moreover the set $\omega^{N_{0}}$ has a finite number of connected components.

It has to be noted that the assumptions of the above theorem (usually referred to as $L^{\infty}$-QUE) are strong ones. Up to now, except in the one-dimensional case where these assumptions obviously hold, in the multi-dimensional case no domain is known where they are satisfied, and it is one of the deepest open problems in mathematical physics to exhibit such a domain. We are however able to prove that the conclusion of Theorem 3 holds true in a hypercube with Dirichlet boundary conditions when one considers the usual Hilbert basis made of products of sine functions, although QUE is not satisfied in such a domain (see [17]).

The theorem says that, for the problem of maximizing $J_{\sigma, N}$ over $\mathcal{U}_{L}$, the sequence of optimal sets $\omega^{N}$ is stationary whenever $L$ is large enough, and $\omega^{N_{0}}$ is then the (unique) optimal set, solution of the problem of maximizing $J_{\sigma}$. It can be noted that the lower threshold in $L$ depends on the 
chosen weights, and the numerical simulations that we will provide indicate that this threshold is sharp in the sense that, if $L<\sigma_{1}$ then the sequence of maximizing sets loses its stationarity feature.

As a conclusion, this weighted version of our spectral criterion can be viewed as a remedy for the spillover phenomenon. Note that, of course, other more evident remedies can be discussed, such as the search of an optimal domain among a set of subdomains sharing nice compactness properties (such as having a uniform perimeter or BV norm), however our aim is here to investigate domains as general as possible (only measurable) and rather to discuss the mathematical, physical and practical relevance of the criterion encoding the notion of optimal observability.

Let us finally note that all our results hold for wave and Schrödinger equations on any open bounded connected subset of a Riemannian manifold (then replacing $\triangle$ with the Laplace-Beltrami operator), with various possible boundary conditions (Dirichlet, Neumann, mixed, Robin) or no boundary conditions in case the manifold is compact without boundary. The abstract framework and generalizations are described in details in [17].

\section{THE CASE OF PARABOLIC PDE'S}

Instead of dealing with a general parabolic model, for the sake of simplicity we consider the heat equation

$$
\partial_{t} y-\triangle y=0, \quad(t, x) \in(0, T) \times \Omega,
$$

with Dirichlet boundary conditions. For any measurable subset $\omega$ of $\Omega$, we observe the solutions of (6) restricted to $\omega$ over the horizon of time $[0, T]$, that is, we consider the observable $z(t, x)=\chi_{\omega}(x) y(t, x)$, where $\chi_{\omega}$ denotes the characteristic function of $\omega$.

For a given measurable subset $\omega$ of $\Omega$, the heat equation (6) is said observable on $\omega$ in time $T$ whenever there exists $C>0$ such that

$$
C \int_{\Omega} y(T, x)^{2} d x \leqslant \int_{0}^{T} \int_{\omega} y(t, x)^{2} d x d t
$$

for every solution of (6) such that $y(0, \cdot) \in \mathcal{D}(\Omega)$. It is well known that, if $\Omega$ is $C^{2}$ or rectangular then this observability inequality holds (see [21]). The observability constant $C_{T}\left(\chi_{\omega}\right)$ is defined as the largest possible constant such that (7) holds, that is the infimum of the quantities $\int_{0}^{T} \int_{\omega} y(t, x)^{2} d x d t / \int_{\Omega} y(T, x)^{2} d x$ over all $y(0, \cdot) \in$ $\mathcal{D}(\Omega) \backslash\{0\}$. As before, randomizing the initial data leads to the definition of the randomized observability constant

$$
C_{T, \text { rand }}\left(\chi_{\omega}\right)=\inf _{j \in \mathbb{N}^{*}} \frac{e^{2 \lambda_{j} T}-1}{2 \lambda_{j}} \int_{\omega} \phi_{j}(x)^{2} d x,
$$

with the significant difference that every integral is multiplied by the weight $\frac{e^{2 \lambda_{j} T}-1}{2 \lambda_{j}}$. This implies completely different results. As before, instead of considering as a criterion the deterministic observability constant, we find more relevant to model the problem of best observation domain as that of maximizing the functional $C_{T \text {,rand }}\left(\chi_{\omega}\right)$ over the set $\mathcal{U}_{L}$.
Theorem 4 ([19]): Assume that $\partial \Omega$ is piecewise $C^{1}$. There exists a unique optimal observation set $\omega^{*}$, solution of the problem of maximizing the functional $C_{T \text {,rand }}\left(\chi_{\omega}\right)$ over the set $\mathcal{U}_{L}$. Moreover, $C_{T}\left(\chi_{\omega^{*}}\right)<C_{T \text {, rand }}\left(\chi_{\omega^{*}}\right)$.

Here, it is understood that the optimal set $\omega^{*}$ is unique within the class of all measurable subsets of $\Omega$ quotiented by the set of all measurable subsets of $\Omega$ of zero measure.

Note that this existence and uniqueness result holds for every orthonormal basis of eigenfunctions of the DirichletLaplacian, but the optimal set depends on the specific choice of the Hilbert basis.

It is remarkable that the optimal observation set $\omega^{*}$ can be built from a finite-dimensional spectral approximation, by keeping only a finite number of modes. The precise result is the following.

Theorem 5 ([19]): For every $N \in \mathbb{N}^{*}$, there exists a unique set $\omega^{N}$ such that $\chi_{\omega^{N}} \in \mathcal{U}_{L}$ maximizes the functional

$$
\chi_{\omega} \mapsto \inf _{1 \leqslant j \leqslant N} \frac{e^{2 \lambda_{j} T}-1}{2 \lambda_{j}} \int_{\omega} \phi_{j}(x)^{2} d x
$$

over $\mathcal{U}_{L}$. Moreover $\omega^{N}$ is semi-analytic, and thus, in particular, it has a finite number of connected components. Furthermore, the sequence of optimal sets $\omega^{N}$ is stationary, and there exists $N_{0} \in \mathbb{N}^{*}$ such that $\omega^{N}=\omega^{*}$ if $N \geqslant N_{0}$.

In particular, it follows that the optimal set $\omega^{*}$ of Theorem 4 is semi-analytic as well and hence has a finite number of connected components. This property is in strong contrast with the results established for wave and Schrödinger equations. For the latter equations, the fact that all frequencies have the same weight causes a strong instability of the optimal sets $\omega^{N}$, in particular the spillover phenomenon mentioned previously. For parabolic equations this instability phenomenon does not occur, and the sequence of maximizers $\omega^{N}$ is constant as soon as $N$ is large enough, equal to $\omega^{*}$. This property is of particular interest in view of designing the best observation set $\omega^{*}$ in practice.

On Figure 2, we compute the optimal domain $\omega^{N}$ in the case $\Omega=(0, \pi)^{2}, L=0.2$ and $T=0.05$, for $N=1, \ldots, 6$. We can observe the expected stationarity property of the sequence of optimal domains $\omega^{N}$ from $N=4$ on (i.e., 16 eigenmodes).
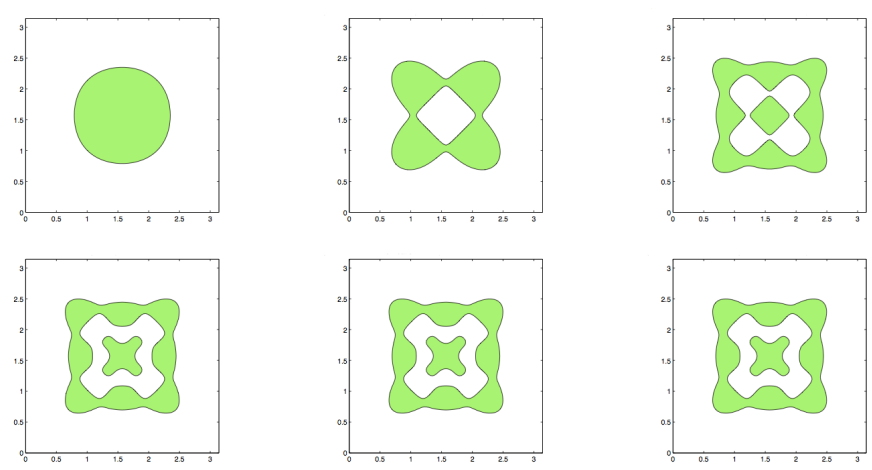

Fig. 2.

These results can be established as well for more general parabolic equations (see [19]), involving in particular 
anomalous diffusion equations and Stokes equations. Let us mention in particular an interesting feature occuring for the anomalous diffusion equation $\partial_{t} y+(-\triangle)^{\alpha} y=0$ in $\Omega$, where $(-\triangle)^{\alpha}$ is some positive power of the Dirichlet-Laplacian, with arbitrary boundary conditions implying $y_{\mid \partial \Omega}=0$. It is proved in [19] that:

- in the Euclidean square $\Omega=(0, \pi)^{2}$, when considering the usual Hilbertian basis of eigenfunctions consisting of products of sine functions, for every $\alpha>0$ there exists a unique optimal set (as in Theorem 4), which is moreover open and semi-analytic;

- in the Euclidean disk $\Omega=\left\{x \in \mathbb{R}^{2} \mid\|x\|<1\right\}$, when considering the usual Hilbertian basis of eigenfunctions parametrized in terms of Bessel functions, we have the following surprising result:

- if $\alpha>1 / 2$ then there exists a unique optimal set (as in Theorem 4), which is moreover semi-analytic and thus has a finite number of connected components;

- if $\alpha<1 / 6$ then any optimal set must have an infinite number of connected components.

\section{CONCLUSION}

We have presented our results for the optimal shape and placement of sensors. Similar results can be derived for actuators (see [16], [17], [19]). Our main contribution is to have highlighted a precise relation between those kinds of shape optimization problems and concentration properties of eigenfunctions (quantum ergodicity issues). Many interesting open questions remain to be investigated, such as:

- Generic nonexistence of an optimal set.

- Other relevant shape optimization problems avoiding the spillover phenomenon.

- Control theoretical consequences for random initial data.

- Optimal stabilization and optimal choice of dampers.

- Optimization of the deterministic observability constant.

- Discrete versions of optimization problems above.

The latter issue might be particularly important in applications both because discrete models are often employed, and also because numerical approximation schemes necessarily end up becoming of discrete nature. In particular, the following type of questions arise as discrete versions of those we have considered in this paper in the continuous setting. Let $A$ be a real symmetric matrix. The problem of the optimal choice of an observation operator $B$ over a certain class $\mathcal{B}$ can be formulated as

$$
\sup _{B \in \mathcal{B}} \inf \left\{\frac{\langle B x, x\rangle}{\|x\|^{2}} \mid \exists \lambda, \quad A x=\lambda x\right\} .
$$

In the context of our paper, $B$ is the (infinite-dimensional) Gramian of observation over $\omega$. But the problem can be generalized in the above more abstract form, allowing us in particular to formulate in an appropriate context the following open question: under which assumptions do the optimal designs commute with discretization schemes?

\section{REFERENCES}

[1] G. Allaire, S. Aubry, F. Jouve, Eigenfrequency optimization in optimal design, Comput. Methods Appl. Mech. Engrg. 190 (2001), 3565-3579.

[2] G. Allaire, A. Münch, F. Periago, Long time behavior of a two-phase optimal design for the heat equation, SIAM J. Control Optim. 48 (2010), no. 8, 5333-5356.

[3] A. Armaoua, M. Demetriou, Optimal actuator/sensor placement for linear parabolic PDEs using spatial $H^{2}$ norm, Chemical Engineering Science 61 (2006), 7351-7367.

[4] C. Bardos, G. Lebeau, J. Rauch, Sharp sufficient conditions for the observation, control, and stabilization of waves from the boundary, SIAM J. Control Optim. 30 (1992), no. 5, 1024-1065.

[5] J.C. Bellido, A. Donoso, An optimal design problem in wave propagation, J. Optim. Theory Appl. 134 (2007), 339-352.

[6] G. Chen, S.A. Fulling, F.J. Narcowich, S. Sun, Exponential decay of energy of evolution equations with locally distributed damping, SIAM J. Appl. Math. 51 (1991), no. 1, 266-301.

[7] F. Fahroo, K. Ito, Optimum damping design for an abstract wave equation, New directions in control and automation, I (Limassol, 1995), Kybernetika (Prague) 32 (1996), no. 6, 557-574.

[8] T.J. Harris, J.F. Macgregor, J.D. Wright, Optimal sensor location with an application to a packed bed tubular reactor, AIChE Journal 26 (1980), no. 6, 910-916.

[9] P. Hébrard, A. Henrot, Optimal shape and position of the actuators for the stabilization of a string, Syst. Cont. Letters 48 (2003), 199-209.

[10] P. Hébrard, A. Henrot, A spillover phenomenon in the optimal location of actuators, SIAM J. Control Optim. 44 2005, 349-366.

[11] S. Kumar, J.H. Seinfeld, Optimal location of measurements for distributed parameter estimation, IEEE Trans. Autom. Contr. 23 (1978), 690-698.

[12] K. Morris, Linear-quadratic optimal actuator location, IEEE Trans. Automat. Control 56 (2011), no. 1, 113-124.

[13] A. Münch, P. Pedregal, Numerical null controllability of semi-linear ID heat equations : fixed point, least squares and Newton methods, Math. Control Relat. Fields 3 (2012), no. 2, 217-246.

[14] A. Münch, F. Periago, Optimal distribution of the internal null control for the 1D heat equation, J. Diff. Equations 250, 95-111 (2011).

[15] Y. Privat, E. Trélat, E. Zuazua, Optimal observation of the onedimensional wave equation, J. Fourier Anal. Appl. 19 (2013), no. 3, 514-544.

[16] Y. Privat, E. Trélat, E. Zuazua, Optimal location of controllers for the one-dimensional wave equation, to appear in Ann. Inst. H. Poincaré Anal. Non Linéaire (2013).

[17] Y. Privat, E. Trélat, E. Zuazua, Optimal observability of the multidimensional wave and Schrödinger equations in quantum ergodic domains, Preprint Hal (2013).

[18] Y. Privat, E. Trélat, E. Zuazua, Complexity and regularity of maximal energy domains for the wave equation with fixed initial data, Preprint Hal (2013).

[19] Y. Privat, E. Trélat, E. Zuazua, Optimal shape and location of sensors or controllers for parabolic equations with random initial data, Preprint Hal (2013).

[20] O. Sigmund, J.S. Jensen, Systematic design of phononic band-gap materials and structures by topology optimization, R. Soc. Lond. Philos. Trans. Ser. A Math. Phys. Eng. Sci. 361 (2003), no. 1806, 1001-1019.

[21] M. Tucsnak, G. Weiss, Observation and control for operator semigroups, Birkhäuser Verlag, Basel (2009).

[22] D. Ucinski, M. Patan, Sensor network design fo the estimation of spatially distributed processes, Int. J. Appl. Math. Comput. Sci. 20 (2010), no. 3, 459-481.

[23] M. van de Wal, B. Jager, A review of methods for input/output selection, Automatica 37 (2001), no. 4, 487-510.

[24] A. Vande Wouwer, N. Point, S. Porteman, M. Remy, An approach to the selection of optimal sensor locations in distributed parameter systems, Journal of Process Control 10 (2000), 291-300. 